\title{
A New Axiomatization of Portfolio Theory
}

\author{
Jan P. H. van Santen \\ Department of Psychology, The University of Michigan, Ann Arbor, Michigan 48109
}

An axiomatization proposed by Coombs (1974) is shown to be insufficient for the representation of gambles in a risk $\times$ expected value space as described by Portfolio Theory. A new axiom system is given which is necessary and sufficient for this representation, and which implies the old axiomatization.

\section{INTRODUCTION}

Portfolio Theory (PT) is a theory specifying how risk and expected value affect preference in the domain of gambles. Since its first appearance (Coombs, 1969), several formulations have been given, both formal (e.g., Coombs, 1974) and informal (e.g., Coombs \& Huang, 1970).

The crux of the theory is the structure in Fig. 1, which states that indifference curves (i.e., connected sets of points $(x, y)$ where $x$ is the expected value and $y$ some risk measure of a gamble) are increasing above a line called $I$, the ideal crest, decreasing below $I$, and are nonintersecting. It is the representability of a preference ordering by this structure that has been tested empirically and for which axioms have been constructed. The reason for constructing a new axiom system is that, as will be shown below, no existing axiomatization states sufficient conditions for the representability of a preference ordering by the structure in Fig. 1.

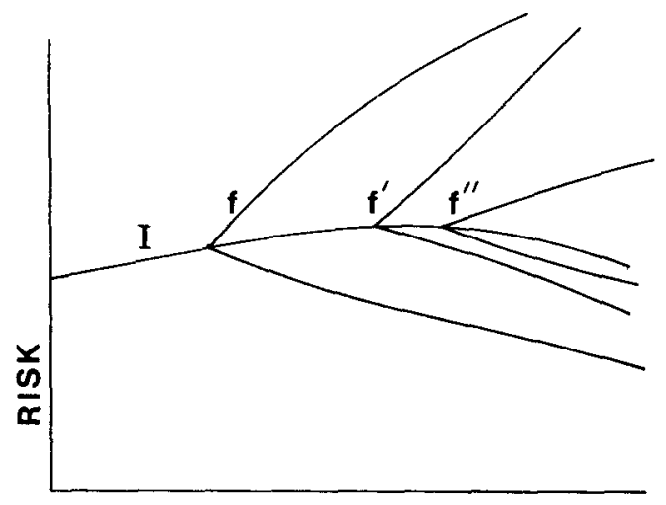

EXPECTED VALUE

FIG. 1. Indifference curves $\left(f, f^{\prime}\right.$, and $\left.f^{\prime \prime}\right)$ and ideal crest $(I)$ according to the Portfolio Theory. 


\section{The Representation Probi.fm}

Definition 1 formalizes the structure in Fig. 1.

Definition 1. $\quad F, I\rangle$ is a Folded Trade-off Structure (FTS) iff

(1) $I$ is a continuous real-valued function on a closed interval $[s, t]$ of $\mathrm{Re}$, the set of real numbers.

(2) $F$ is a nonempty set of binary relations on $\mathrm{Re}$, such that for all $f, f^{\prime} \in F$ :

(a) If $f \neq f^{\prime}$ then $f \cap f^{\prime}=\varnothing$.

(b) $f \cap I$ contains exactly one element, denoted $\left(a_{f}, b_{f}\right)$.

(c) Let $f^{*}$ be the converse of relation $f$ (i.e., $f^{*}=\{(y, x) \mid(x, y) \in f\}$ ), and let $L_{f}$ and $U_{f}$ be real numbers such that $L_{f}<b_{f}<U_{f}$. Then $f^{*}$ is strictly decreasing on $\left[L_{f}, b_{f}\right]$, strictly increasing on $\left(b_{f}, U_{f}\right]$, and continuous on $\left[L_{f}, U_{f}\right]$.

The graph of any $f$ in $F$ is an indifference curve in expected value $\times$ risk coordinates; $f^{*}(y)=x$ iff $x$ is the unique expected value at which risk $y$, combined with $x$, is on the indifference curve; and $f^{*}$ attains its minimum, $a_{f}$, at risk $=b_{f}$, the point $\left(a_{f}, b_{f}\right)$ being on the ideal crest.

Definition 2. Let $\langle F, I\rangle$ be a FTS, let $\mathbf{x}, \mathbf{y} \in \bigcup_{f \in F} f$, and let $f$ be the (unique) curve on which $\mathbf{x}$ lies, and $f^{\prime}$ the (unique) curve on which $\mathbf{y}$ lies. Then define $\mathbf{x} \geqslant_{F} \mathbf{y}$ iff $a_{f} \geqslant a_{f^{\prime}}$.

Definition 3. A set $G$ with three relations $\succcurlyeq_{r}, \succcurlyeq_{e v}$, and $\succcurlyeq_{p}$ is FTS-representable iff there exist real-valued functions $R$ and $E$ on $G$, and a FTS $\langle F, I\rangle$ such that for all $a, b \in G$ :

(1) $(E(a), R(a)) \in \bigcup_{f \in F} f$.

(2) $a \succcurlyeq_{p} b$ iff $(E(a), R(a)) \geqslant_{F}(E(b), R(b))$.

(3) $a \geqslant_{e v} b$ iff $E(a) \geqslant E(b)$, and $a \geqslant_{r} b$ iff $R(a) \geqslant R(b)$.

The three relations on $G$ are to be interpreted as a risk, expected value, and preference ordering. In words, Definition 3 states that these relations are FTS-representable if we can represent the elements of $G$ as points in an expected value $\times$ risk space, and can draw curves through these points such that the resulting structure is a FTS with indifference curves representing more attractive gambles when going to the right. In what follows, we will denote the symmetric, respectively asymmetric, parts of $\succcurlyeq_{r}$ by $\sim_{r}$, respectively $>_{r}$. Similarly for $\succcurlyeq_{e v}, \succcurlyeq_{D}$, and $\geqslant_{F}$.

Given these definitions, it is easy to see why the axioms proposed by Coombs (1974) are insufficient. These axioms are the following:

The structure $\left\langle G, \succcurlyeq_{r}, \succcurlyeq_{p}\right\rangle$ is a Portfolio structure iff there exists for each expectation level $t$ a scale $\phi_{t}$ (interpreted as a risk preference scale) such that: 
1. If $E(A)=E(B)=t$ then $A \geqslant{ }_{p} B$ iff $\phi_{t}(A) \geqslant \phi_{t}(B)$, where $E$ is the expectation operator.

2. If $E(C)=E(B)=E(A)=t$ and $C \geqslant_{r} B \geqslant_{r} A$ then either $\phi_{t}(B) \geqslant \phi_{t}(A)$ or $\phi_{t}(B) \geqslant \phi_{t}(C)$.

3. If $E(A) \geqslant E(B)$ and $\phi_{a}(A) \geqslant \phi_{b}(B)$, where $a=E(A)$ and $b=E(B)$, then $A \succcurlyeq_{p} B$. Moreover, if either inequality is strict, then $A \succ_{p} B$. (Coombs, 1974, p. 26).

Consider the following counterexample. Let $G$ be a nonempty finite set of gambles chosen such that there exists a transitive and connected ordering $\succcurlyeq_{r}$ on $G$ such that for some $a_{0}, b_{0} \in G, a_{0} \sim_{r} b_{0}$, and $E\left(a_{0}\right)=1 / E\left(b_{0}\right)>1$. By finiteness of $G$, and because $\geqslant_{r}$ is a weak ordering, there exists a real-valued function $R$ on $G$ such that for all $a, b \in G$, $a \geqslant{ }_{r} b$ iff $R(a) \geqslant R(b)$, and $R\left(a_{0}\right)=R\left(b_{0}\right)=0$. Define for any real $t$ for which the set $G_{t}$, containing all gambles having an expected value of $t$, is nonempty, the function $\phi_{t}: G_{t} \rightarrow \operatorname{Re}$ as follows: $\phi_{t}(a)=1 /(t+|R(a)|)$. Finally, let $a \geqslant_{p} b$ iff $E(a)+\phi_{E(a)}(a) \geqslant$ $E(b)+\phi_{E(b)}(b)$. Clearly, this structure satisfies axioms 1, 2, and 3. However, it is not FTS-representable, because $a_{0} \sim_{p} b_{0}, a_{0} \sim_{r} b_{0}$, and $a_{0}>_{e v} b_{0}$.

The reason why this set is not FTS-representable is that the functions $\phi_{t}$ are defined such that they decrease with expected value if one holds risk constant. All previous axiomatizations and informal presentations of PT have the same weakness in that they do not sufficiently restrict $\succcurlyeq_{p}$ on gambles differing in expected value.

As a concluding remark, it should be pointed out that FTS-representability implies single-peakedness of $\succcurlyeq_{p}$ with respect to $\succcurlyeq_{r}$ at a fixed expected value level, i.e., iff $a \sim_{e v} b \sim_{e v} c$ and $a>_{r} b>_{r} c$ then $b>_{p} a$ or $b>_{p} c$. This follows from the fact that a vertical line through a FTS intersects the indifference curves such that under $I$ the intersections increase with respect to $\geqslant_{F}$ and decrease above $I$.

\section{A New Axiomatization}

We have restricted ourselves to the case where $G$ is finite. Not only does this considerably simplify matters, there also do not seem to exist theoretical or practical reasons for treating the infinite case.

Let $G$ be a finite set with three binary relations $\geqslant_{r}, \geqslant_{e v}$, and $\geqslant_{p}$.

Axiom (i). $\succcurlyeq_{r}, \succcurlyeq_{e v}$, and $\succcurlyeq_{p}$ are weak orders (i.e., transitive and connected).

Let $G / \sim_{e v}=\left\{G_{1}, \ldots, G_{m}\right\}$, and define $G_{i}{ }^{I}=\left\{a \mid a \in G_{i}\right.$ and if $c \in G_{i}$ then $\left.a \succcurlyeq_{p} c\right\}$. Thus, $G_{i}{ }^{l}$ contains the elements maximally preferred in the set $G_{i}$ of all elements having a particular expected value.

Axiom (ii). For all $i=1, \ldots, m$ if $a, b \in G_{i}{ }^{I}$ then there is no $c \in G_{i}$ such that $a>_{r} c>_{r} b$ or $b>_{r} c>_{r} a$.

Note that Axiom (ii) implies that there exist at most two risk levels within $G_{i}{ }^{l}$. The axiom will be used to define two subsets of $G, G^{+}$and $G^{--}$, and it guarantees that all 
gambles belong to either $G^{+}$or $G^{-}$with the exception of the gambles belonging to sets $G_{i}{ }^{I}$ having only one risk level. We will use $G^{I}$ for the union of all sets $G_{i}{ }^{I}$. Let $G^{+}=$ $\left\{a \mid\right.$ if $a \in G_{i}$ then there is $c \in G_{i}{ }^{l}$ such that $\left.a>_{r} c\right\}$. And $G^{-}=\left\{a \mid\right.$ if $a \in G_{i}$ then there is $c \in G_{i}{ }^{l}$ such that $c>_{r} a$ \}. Note that $G^{+} \cap G^{-}=\varnothing$, but not necessarily $G=G^{+} \cup G^{-}$. $G^{+}$or $G^{-}$may have elements in common with $G^{J}$. Finally, $G=G^{+} \cup G^{-} \cup G^{I}$. To make this particular choice of defining $G^{+}$and $G^{-}$understandable the role of ideal gambles in the present axiomatization should be pointed out. Because $G$ is finite, we can never be certain if there do not exist gambles outside $G$ which, if included in the experiment, would be preferred over the maximally preferred gambles in their respective expected value equivalence classes. In other words, being maximally preferred in $G_{i}$ does not mean being ideal in the absolute sense. Now, what we are interested in is obtaining a representation of $G$ that leaves room for those ideal gambles. The definition of $G^{+}$is such that in the representation a possible ideal gamble would have to be represented below the points on which the gambles in $G^{+}$having the same expected value as the ideal gamble are mapped. Similarly for $G^{-}$. In fact, no other definitions of $G^{-}$ and $G^{-}$would have been possible.

Axiom (iii). If $a, b \in G^{+}, a \succcurlyeq_{e v} b$ and $b \succcurlyeq_{r} a$ then $a \geqslant_{\nu} b$. If $a, b \in G^{-}, a \succcurlyeq_{e v} b$ and $a \succcurlyeq_{r} b$ then $a \geqslant_{p} b\left(>_{p}\right.$ if either antecedent inequality is strict).

Axiom (iii) states that for gambles which are too risky $\left(G^{+}\right)$a gamble becomes more attractive when risk decreases and expected value increases, and that the converse holds to $G$.

The three axioms simply translate a FTS into empirical order relations. Consequently, most of the proofs are rather trivial.

THEOREM 1. A set $G$ with three binary relations $\succcurlyeq_{r}, \succcurlyeq_{e v}$, and $\succcurlyeq_{p}$ satisfies Axioms (i)-(iii) iff it is FTS-representable.

Proof (Sufficiency). By (i) there exist scales $E$ and $R$ satisfying Definition 3(3). Let $h(a)=(E(a), R(a))$. Let $[a]=\left(\left\{c \mid c \sim_{e v} a\right\},\left\{d \mid d \sim_{r} a\right\}\right)$, and define $[a] \geqslant *[b]$ iff

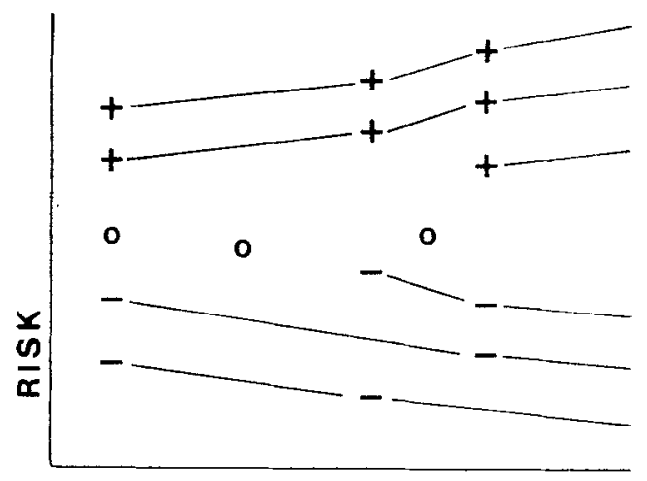

EXPECTED VALUE

Fig. 2. Indifference curves prior to the construction of the ideal crest. Elements of $h\left[G^{+}\right]$are indicated by + , elements of $h\left[G^{-}\right]$by --, and elements of $h\left[G-G^{+}-G^{-}\right]$by $\bigcirc$. 
$a \succcurlyeq_{p} b$. By (iii), $\geqslant_{*}^{*}$ is well defined. Moreover, (iii) implies that $\left\langle G^{+} / \sim_{e v} \times G^{+} / \sim_{r}, \geqslant^{*}\right\rangle$ satisfies independence in the conjoint measurement sense (Krantz, Luce, Suppes, \& Tversky, 1971), and that the induced orderings on the components are isotonic, respectively antitonic, with $\succcurlyeq_{e v}$, respectively $\succcurlyeq_{r}$. Hence we can draw nonintersecting, strictly increasing indifference curves through $h\left[\mathrm{G}^{+}\right]$always terminating right of the rightmost point through which a curve is drawn. Likewise for $h\left[G^{-}\right]$. The result is shown in Fig. 2. Now a curve $I$ can be drawn such that it does not cross any of the existing curves and that it has a unique risk level for every expected value. Note that in case $h\left[G_{i}{ }^{I}\right]$ is one point, $I$ can be drawn at either side of that point. Both for $\left\langle\boldsymbol{G}^{+} / \sim_{e v} \times G^{+} / \sim_{r}, \geqslant *\right\rangle$ and for $\left\langle G^{-} / \sim_{e v} \times G^{-} / \sim_{r}, \geqslant *\right\rangle$ the induced ordering on the first component is isotonic with $\succcurlyeq_{e v}$. Hence we can extend both groups of curves in Fig. 2 to the opposite side of $I$, such that the set of new curves is nonintersecting. $I$ is crossed exactly once by each curve, and every extended curve crosses $I$ at its leftmost point. Clearly, the resulting structure is a FTS satisfying Definitions 3(1)-(3).

(Necessity). Let $s(a)$ be the number of curves left of the curve containing $h(a)$. Then $s(a) \geqslant s(b)$ iff $a \geqslant_{p} b$. Also, $R$ and $E$ are ordinal scales. Thus, $\geqslant_{r}, \geqslant_{e v}$, and $\geqslant_{p}$ must be weak orders. For (ii) assume $a, b \in G_{i}{ }^{l}, a \succ_{r} b\left(a \sim_{r} b\right.$ is trivial) and there is $c \in G_{i}$ such that $a>_{r} c>_{r} b$. Hence $h(c)$ must lie on a vertical line through $h(a)$ and $h(b)$ between these points. Also, there exists $f \in F$ such that it contains both $h(a)$ and $h(b)$. Then $h(c)$ has to lie on a curve right of $f$, which implies $c>_{p} a, b$, contradicting the definition of $G_{i}{ }^{I}$. Before we conclude the proof by showing (iii), first note that (iii) is equivalent to: if $a, b \in G, a \succcurlyeq_{e v} b$ and $b \succcurlyeq_{p} a$ then $a \succcurlyeq_{r} b$, and if $a, b \in G^{-}, a \geqslant_{e v} b$ and $b \succcurlyeq_{p} a$ then $b \succcurlyeq_{r} a$ ( $\succ_{r}$ if either antecendent inequality is strict).

Assume $a, b \in G^{+}, a \geqslant_{e v} b$, and $b \geqslant_{p} a$. Then $E(a) \geqslant E(b)$ and $h(b) \geqslant_{F} h(a)$. If $a \sim_{p} b$ then $h(a)$ and $h(b)$ lie on the same $f \in F$, which implies $R(a) \geqslant R(b)$ by the isotonicity of the part of $f$ above $I$. Assume $b>_{p} a$, and let $h(a) \in f$ for some $f \in F$. Then $h(b)>_{F} h(a)$ together with the continuity of $f *$ imply that a vertical line through $h(b)$ intersects $f$ in some point $(E(b), x)$, where $x>R(b)$. By isotonicity of $f$ we have: $E(a) \geqslant E(b)$ implies $R(a) \geqslant x>R(b)$, and thus $a>_{r} b$. Likewise we can show that $a>_{e v} b$ and $b \succcurlyeq_{p} a$ imply $a>_{r} b$. The proof of the second part of (iii) is similar to that of the first part and will be omitted.

The uniqueness of this representation is difficult to describe. Clearly, any strictly increasing continuous functions preserve the representation. However, given two FTSs representing the same empirical system, there do not necessarily exist strictly increasing functions transforming one FTS into the other, because $I$ is not necessarily monotonic.

\section{Discussion}

The present axiomatization differs from the system proposed by Coombs (Coombs, 1974) not only in that it states necessary and sufficient conditions, but also in that the primitive terms are different: $\phi_{t}$ is eliminated and $E$ is replaced by $\succcurlyeq_{e v}$. It would be of interest to investigate whether or not the previous system is implied by the present 
system. In order to do so, both $E$ and $\phi_{t}$ have to be defined in terms of three binary relations satisfying Axioms (i)-(iii). The definition of $E$ is straightforward: By $i$ there exists a scale $E$ such that $E(a) \geqslant E(b)$ iff $a \geqslant_{e v} b$. For $\phi_{t}$ the situation is more complicated. For example, $\phi_{t}$ cannot be defined as the restriction of an ordinal preference scale $\phi$ (which exists, by (i)) to the subset $G_{t}$. In that case, axiom 3 would be violated by any $b, c$ for which $E(b)>E(c)$ and $\phi_{E(b)}(b)=\phi_{E(c)}(c)$, because the second antecedent implies $a \sim{ }_{p} b$, whereas the strictness clause in axiom 3 implies $a \geqslant_{p} b$. However, if we define $\phi_{t}$ as follows, the desired result can be shown. First, consider the following relations on $G$ :

$$
\begin{aligned}
R & =\left\{(a, b) \mid E(b) \geqslant E(a) \text { and } a \succcurlyeq_{p} b\right\} \\
R^{-} & =\left\{(a, b) \mid E(b)=E(a) \text { and } a \sim_{p} b\right\} \\
R^{>} & =R-R^{=} .
\end{aligned}
$$

$R, R^{-}$, and $R^{>}$are transitive but not necessarily connected. If we define $\phi(a)$ as the number of elements $b$ such that $a R>b$, then $\phi$ satisfies: if $a R^{=} b$ then $\phi(a)=\phi(b)$ and if $a R>b$ then $\phi(a)>\phi(b)$. Then let $\phi_{t}$ be the restriction of $\phi$ to $G_{t}$. Now we can show:

THEOREM 2. If a finite set $G$ with three binary relations $\succcurlyeq_{e v}, \succcurlyeq_{r}$, and $\succcurlyeq_{p}$ satisfies Axioms (i)-(iii) then real-valued functions $E$ and $\phi_{t}$ can be defined on $G$ such that axioms 1-3 hold.

Proof. Let $E(a)=E(b)=t$ and $a \geqslant_{p} b$, then $a R b$ and thus $\phi_{t}(a) \geqslant \phi_{t}(b)$. If $b \succ_{p} a$ then $b R^{>} a$ and thus $\phi_{t}(b)>\phi_{t}(a)$. Let $E(a)=E(b)=E(c)=t$, and let $c \geqslant_{r} b \geqslant_{r} a$. There are three cases:

(1) $b \in G^{+}$, then $c \in G^{+}$and by (iii), $b \geqslant_{p} c$.

(2) $b \in G^{-}$, then $a \in G^{-}$and by (iii), $b \succcurlyeq_{p} a$.

(3) $b \in G^{I}$, then $b \succcurlyeq_{p} a, c$ by definition of $G^{I}$.

Axiom 3 follows immediately from the definition of $\phi_{l}$.

This shows that indeed (1)-(3) state necessary conditions for FTS-representability.

The main reason for not using $\phi_{t}$ as a primitive term in the present axiomatization is that it unnecessarily complicates matters, as the simplicity of the new axioms derives from the fact that this system is a direct translation of a FTS into empirical order relations. A similar argument might be given for replacing $E$ by $\succcurlyeq_{e v}$. However, there are two more reasons. First, properties of expectation, like being a linear operator, are irrelevant for FTS-representability. The only property relevant is that in terms of $E$ a weak ordering over $G$ can be defined. More important is the following theoretical consideration. From a psychological point of view, it is not at all obvious that a gamble is perceived in terms of (risk and) expected value. In fact, it would be rather surprising if individuals were able to correctly order gambles according to expected value, even when given the most explicit instructions specifying the meaning of expected value. In that case, PT formulated in terms of expected value is incorrect. By formulating the theory to allow for biases in the perception of expected value this problem is circumvented. 


\section{ACKNOWLEDGMENT}

I want to thank David H. Krantz for suggesting some changes in the axioms and for editorial comments on the write-up.

\section{REFERENCES}

Coombs, C. H. Portfolio theory: A theory of risky decision making. La Decision. Centre National de la Recherche Scientifique. Paris: 1969.

Coomss, C. H. Portfolio theory and the measurement of risk. Michigan Mathematical Psychology

Program technical report 1974-16.

Coombs, C. H., \& HuANG, L. C. Tests of a portfolio theory of risk preference. Journal of Experimental Psychology, 1970, 85, 23-29.

Krantz, D. H., Luce, R. D., Suppes, P., \& Tversky, A. Foundations of measurement, Vol. I. New York: Academic Press, 1971.

ReCeIved: January 26, 1977 